\title{
Rumenostomia com colocação de cânula flexível em ovinos
}

\author{
Rumenostomy with placement of flexible cannula in sheep \\ Rafael Stedile ${ }^{1}$, Carlos Afonso de Castro Beck' ${ }^{1}$, Fernanda Silveira Nóbrega ${ }^{2}$, Antônio de Pádua Ferreira \\ da Silva Filho', Márcio Poletto Ferreira', Marcelo Meller Alievi', Fabiana Schiochet' ${ }^{1}$, Ângela Ilha Bordin ${ }^{3}$, \\ Paula Cristina Gonzalez ${ }^{4}$ \& Manoela Lampert ${ }^{4}$
}

\begin{abstract}
RESUMO
A colocação de cânulas no sistema digestório é freqüentemente requisitada para estudos experimentais de digestibilidade, principalmente em animais de produção. A colocação de cânulas impede o extravasamento de gás e conteúdo ruminal durante os intervalos de coleta, sendo utilizada em várias espécies e porções do trato digestório. $\mathrm{O}$ objetivo deste trabalho é a descrição de uma técnica cirúrgica de rumenostomia com colocação de cânula flexível de borracha em ovinos. Foram utilizados 14 ovinos machos inteiros adultos hígidos destinados a experimentação em nutrição de ruminantes. Após sedação e anestesia local, os animais foram submetidos à rumenostomia com colocação de cânulas flexíveis de borracha. A técnica se mostrou adequada para produção da fístula, apenas um dos animais apresentou extravasamento em torno da cânula após a implantação da mesma. A rumenostomia para implantação da cânula flexível é de fácil realização e ajuste anatômico em ovinos, proporcionando boa adaptação dos animais.
\end{abstract}

Descritores: fístula ruminal, rúmen, sistema digestório, cânula de borracha.

\section{ABSTRACT}

The placement of cannula in the digestive system is frequently requested for experimental studies of digestibility, mainly in farm animals. The placement of cannula avoids the leakage of gas and rumen content during the collection intervals, being used in several species and portions of the digestive system. The objective of this study is to describe a surgical technique of rumenostomy with placement of flexible rubber cannula in sheeps. Fourteen healthy adult male sheeps destined to the experimentation in nutrition of ruminant were used. After sedation and local anesthesia, the animals were submitted to the rumenostomy with placement of flexible rubber cannula. The technique was appropriate for the fistulae producing, and just one of the animals presented leakage around the cannula after the implantation of it. The rumenostomy for implantation of the flexible cannula is a simple method and produces appropriate anatomical adjustment in sheeps, providing good adaptation of the animals.

Key words: ruminal fistulae, rumen, digestive system, rubber cannula.

${ }^{1}$ Faculdade de Veterinária, Universidade Federal do Rio Grande do Sul (UFRGS). ${ }^{2}$ Residente do Hospital de Clínicas Veterinárias (HCV)UFRGS. ${ }^{3}$ Doutoranda, Programa de Pós-graduação em Medicina Veterinária, Universidade Federal de Minas Gerais (UFMG). ${ }^{4}$ Acadêmica de Medicina Veterinária - UFRGS. CORRESPONDÊNCIA: R. Stedile [stediler@bol.com.br]. 


\section{INTRODUÇÃO}

A colocação de cânulas no sistema digestório é freqüentemente requisitada para estudos experimentais de digestibilidade, principalmente em animais de produção. A colocação de cânulas impede o extravasamento de gás e conteúdo ruminal durante os intervalos de coleta, sendo utilizada em várias espécies e porções do trato digestório. A rumenostomia com colocação de cânulas também é utilizada como auxiliar no tratamento de afecções do trato digestório de ruminantes [4]. Existem descrições de técnicas cirúrgicas em ovinos de colocação de cânulas em rúmen [5], abomaso [6], duodeno e ceco de ovinos [3]. Nos estudos citados acima foram utilizadas cânulas rígidas, geralmente de policloreto de vinila (PVC). O objetivo deste trabalho é a descrição de uma técnica cirúrgica de rumenostomia com colocação de cânula flexível de borracha em ovinos.

\section{MATERIAIS E MÉTODOS}

Foram utilizados 14 ovinos machos inteiros adultos, oriundos do Laboratório de Zootecnia da Faculdade de Agronomia da Universidade Federal do Rio Grande do Sul (UFRGS), destinados a experimentação em nutrição de ruminantes. Os procedimentos foram realizados no Hospital de Clínicas
Veterinárias (HCV) da UFRGS. Após jejum de 24 horas, os animais foram tranquiilizados com maleato de acepromazina ${ }^{1} 0,1 \mathrm{mg} / \mathrm{kg}$ por via intramuscular. Após 20 minutos, os carneiros eram posicionados em decúbito lateral direito, sendo realizada tricotomia ampla na região da fossa paralombar esquerda. Utilizou-se lidocaína ${ }^{2} 1 \%$ (sem vasoconstritor) no bloqueio paravertebral associado à infiltração local na linha de incisão e instilação sobre o peritônio e na camada serosa do rúmen. $\mathrm{Na}$ anti-sepsia foi utilizada a sequiência álcool, polivinilpirrolidona iodo (PVPI) ${ }^{3}$ e álcool. Em seguida, foi realizada incisão de pele de aproximadamente $9 \mathrm{~cm}$ na região central da fossa paralombar esquerda. Os músculos oblíquo externo, oblíquo interno e transverso do abdome, foram incisados na sequiência. Realizou-se a identificação e secção do peritônio. Para facilitar a sutura, optouse por fixar o peritônio temporariamente com uma pinça de Allis (Figura $1 \mathrm{~A}$ ). A redução do espaço morto, ao redor da cânula da fáscia externa do músculo oblíquo externo e peritônio, foi feita com ácido poliglicólico ${ }^{4} 2-0$, no padrão de sutura contínuo simples (Figura $1 \mathrm{~B}$ ). Em seguida, realizou-se exposição e fixação do rúmen na pele com categute ${ }^{5}$ n.1 com padrão de sutura contínuo simples (Figura $1 \mathrm{C}$ ),

Figura 1. Rumenostomia com colocação de cânula flexível de borracha em ovinos. A - Fixação do peritônio e fáscia muscular do transverso abdominal para exposição do rúmen. $\mathbf{B}$ - Aproximação do peritônio e fáscia externa do músculo obliquo externo. $\mathbf{C}$ - Fixação do rúmen à pele. D - Remoção elíptica da parede ruminal. E - Eversão e dobradura da cânula. F - Cânula flexível inserida na fístula ruminal.

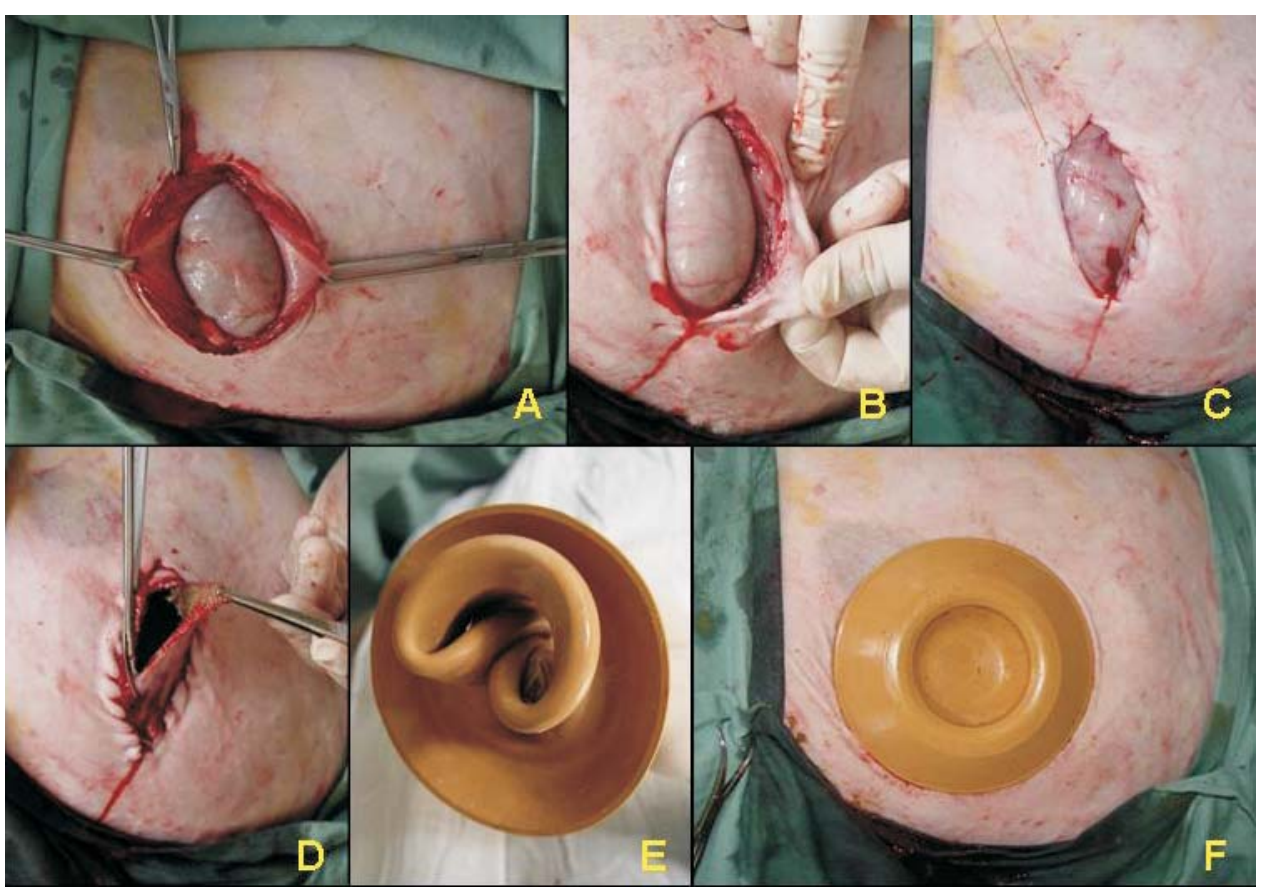


incisão elíptica a aproximadamente $3 \mathrm{~mm}$ da linha de fixação (Figura $1 \mathrm{D}$ ). A cânula foi submersa em solução de PVPI a $5 \%$ por 30 minutos, sendo lavada com solução fisiológica estéril para colocação. A cânula é evertida e dobrada para a rumenostomia, sendo imediatamente fechada com a tampa de vedação (Figura 1 E). Utilizou-se cânula flexível de borracha com diâmetro do disco externo de $15 \mathrm{~cm}$, disco interno $13 \mathrm{~cm}$ e abertura útil da cânula com $7,5 \mathrm{~cm}$, tendo $3,5 \mathrm{~cm}$ de espessura (Figura $1 \mathrm{~F}$ ). No pós-operatório, foi administrado flunixin meglumine ${ }^{6} 2 \mathrm{mg} / \mathrm{kg}$, por via intramuscular, a cada 24 horas por três dias. A terapia antimicrobiana à base de penicilina com estreptomicina ${ }^{7}$ em dose única.

\section{RESULTADOS}

A utilização de tranqüilização com maleato de acepromazina, associada à anestesia local com lidocaína, foi adequada para realização do procedimento. O tempo de cirurgia variou entre 15 e 25 minutos. No pós-operatório imediato, os animais apresentaram posição quadrupedal e alimentaramse normalmente.

A técnica se mostrou adequada para produção da fístula, apenas um dos animais apresentou extravasamento em torno da cânula, após a implantação da cânula, sendo solucionado com aproximação da musculatura da parede abdominal e fixação do rúmen no novo vértice. Esta complicação foi decorrente da maior incisão dos planos realizados neste animal.

\section{DISCUSSÃO}

No presente trabalho, a flexibilidade da cânula de borracha permitiu uma excelente adaptação na rumenostomia em ovinos, o que está de acordo com o observado em estudo com cânulas semelhantes em lhamas [2].

Após seis meses, foi observado pequeno vazamento em quatro animais. O extravasamento de conteúdo é citado como uma das complicações da fístula [7]. Além do aspecto higiênico, o extravasamento pode contribuir pra debilitar o animal, contudo, os animais do presente trabalho encontravam-se em boa condição corporal, sem alteração significativa do processo fermentativo ruminal. Estes achados são semelhantes ao encontrado em outros estudos em ovinos e alpacas [1,5]. Outra complicação citada é a necrose da musculatura nas proximidades da cânula [8]. A rumenopexia evitou a contaminação da cavidade abdominal. Não houve sangramento significativo durante a incisão dos planos musculares. Não foi evidenciada necrose em nenhum dos casos do estudo. Dois animais foram a óbito no pós-operatório com sete e trinta dias, sendo que o primeiro animal apresentou sinais compatíveis com tétano, não sendo identificado o local de infecção. Não havia sinais de peritonite, encontrando-se o local da rumenopexia íntegro. Decorrido os seis meses, a cânula ainda apresentava boa flexibilidade, sem sinais de ressecamento, fragilidade ou alterações conformacionais.

\section{CONCLUSÕES}

A rumenostomia para implantação da cânula flexível é de fácil realização e ajuste anatômico, proporcionando boa adaptação dos animais. A fístula foi efetiva no seu objetivo, permitindo a coleta de material durante a experimentação sem grande comprometimento da qualidade de vida do animal.

\section{NOTAS INFORMATIVAS}

${ }^{1}$ Acepran 1\%, Univet, Rua Padre José, 131, Mogi Mirim, SP.

${ }^{2}$ Lidovet, Bravet, Rua Visconde de Santa Cruz, 276, Rio de Janeiro, RJ.

${ }^{3}$ Asteriodine - P.V.P.I Tópico, Aster, Av. independência, 2541, Sorocaba, SP.

${ }^{4}$ Ácido Poliglicólico PGA Violeta Bioline/Brasuture, Av. Visconde de Guarapuava, 3577, Curitiba, PR.

${ }^{5}$ Catgut cromado 1 - Suturbras Indústrias e Comércio Ltda, Penha, 129, São Paulo, SP.

${ }^{6}$ Banamine ${ }^{\circledR}$ injetável, Schering-Plough Saúde Animal Indústria e Comércio Ltda. - Avenida Sir Henry Wellcome, 335, Cotia, SP

${ }^{7}$ Agrodel, Jofadel Produtos Veterinários, Av. Dr. José da Frota Vasconcelos, 100, Varginha, MG.

\section{REFERÊNCIAS}

1 Cabrera C.R., López V.A., Maiztegui V.J. \& Marín G.M.P. 1996. Fistulación y canulación permanente del comportamiento 1 (rumen) en alpacas (Lama pacos). Avances en Medicina Veterinaria. 11: 108-111. 
Stedile R., Beck C.A.C., Nóbrega F.S., Filho A.P.F.S., Ferreira M.P., Alievi M.M., Schiochet F., Bordin A.I., Gonzalez P.C. \& Lampert M. Rumenostomia com colocação de cânula flexível em ovinos. Acta Scientiae Veterinariae. 36(1):35-38.

2 Cabrera R., López A., Morales S., Salazar H. \& Fuentes A.M. 2000. Fistulación y canulación permanente del compartimento 1 (rumen) en Llamas (Lama glama). Archivos de Medicina Veterinária. 32: 131-138.

3 Dougherty R. W. 1955. Permanent stomach and intestinal fistulas in ruminates: some modifications and simplifications. Cornell Veterinarian. 45: 331-360.

4 Miguel M.C.V., Lopes M.A.F., Ribeiro Filho J.D. \& Boges M.C.B. 2004. Rumenostomia com a implantação de uma cânula flexível numa novilha com disfagia. In: Anais do Congresso Paulista de Medicina Veterinária (Santos, Brasil). 1 CD-ROM.

5 Silva Filho A.P.S. \& Beck C.A.C. 1990. Comparação entre duas técnicas de rumenostomia com colocação de cânulas de PVC, em ovinos. Arquivos da Faculdade de Veterinária da UFRGS. 18: 103-106.

6 Silva Filho A.P.S., Leão M. I. \& Beck C.A.C. 1990. Abomasostomia com colocação de cânula de PVC em ovinos. Arquivos da Faculdade de Veterinária da UFRGS. 18: 107-109.

7 Thyfault H.A., Leffel E. C. \& Huang M.D. 1975. Simplified method for producing permanent ruminal fistulae. Journal of Dairy Science. 58: 1899-1901.

8 Yarns D.A. \& Putnam P.A. 1962. An economical rumen cannula. Journal of Animal Science. 21: 744. 